TITLE:

\title{
Studies on monodromy preserving deformation of linear differential equations on elliptic curves
}

$\operatorname{AUTHOR}(S)$ :

Mano, Toshiyuki

\section{CITATION:}

Mano, Toshiyuki. Studies on monodromy preserving deformation of linear differential equations on elliptic curves. Journal of Mathematical Physics 2009, 50(10): 103501.

ISSUE DATE:

2009-10

URL:

http://hdl.handle.net/2433/87414

RIGHT:

c 2009 American Institute of Physics. 


\title{
Studies on monodromy preserving deformation of linear differential equations on elliptic curves
}

\author{
Toshiyuki Mano a) \\ Research Institute for Mathematical Sciences, Kyoto University, Kyoto 606-8502, Japan
}

(Received 15 February 2009; accepted 23 July 2009; published online 1 October 2009)

\begin{abstract}
We study a monodromy preserving deformation (MPD) of linear differential equations on elliptic curves. As the first of our results, we describe asymptotic behaviors of solutions to the MPD system when the elliptic curve degenerates to a rational curve. As the second, we find explicit solutions for special values of parameters where the MPD system is linearizable. Our solutions are written in terms of integrals of theta functions. We also show that they converge to the hypergeometric functions applying the above asymptotic formula when the elliptic curve degenerates to a rational curve. (C) 2009 American Institute of Physics.
\end{abstract}

[doi:10.1063/1.3204973]

\section{INTRODUCTION}

Monodromy preserving deformations (MPDs) of linear differential equations with rational coefficients yield many interesting nonlinear special functions such as the Painlevé transcendents and they have many applications in mathematics and mathematical physics. In this paper, we study the MPD of Fuchsian differential equations with two regular singular points on elliptic curves. Okamoto ${ }^{13,14}$ derived the Hamiltonian systems that govern the MPD of second-order Fuchsian differential equations on an elliptic curve. Although his results are restricted to the deformation with respect to configurations of points on an elliptic curve, in fact, we can consider the deformation with respect to moduli of elliptic curves. Kawai ${ }^{6}$ derived the Hamiltonian system that governs the MPD with respect to the moduli of elliptic curves. Nowadays other formulations for the MPD on elliptic curves have been proposed by several authors. ${ }^{8,19}$ Those formulations might be conceptually equivalent, however, the explicit relationship between them seem not to be clear. In this paper, we mainly follow Korotkin-Samtleben's formulation, ${ }^{8}$ which treats the MPD of rank-two systems of linear differential equations on elliptic curves. We prove in Sec. II that Korotkin-Samtleben's formulation is equivalent to Okamoto-Kawai's formulation which treats second-order single differential equations (Proposition 2.5). This result may be regarded as an analog to the Garnier-Schlesinger correspondence in the rational case. ${ }^{15}$

The main purpose in the present paper is to study detailed properties of solutions to the MPD system on elliptic curves. We treat two aspects of the problem. One is to describe asymptotic behaviors of the solutions when the elliptic curve degenerates to a rational curve. The other is to find explicit solutions to the MPD system for special values of parameters where the MPD system is linearizable. In Sec. III, we study asymptotic behaviors of generic solutions to the MPD system around the boundary in the moduli space of elliptic curves. As is mentioned above, the MPD system on elliptic curves has two types of independent variables, namely, configurations of points on an elliptic curve and moduli of elliptic curves. The fiber of the boundary point forms an irreducible rational curve with one node (which we call a rational nodal curve). In his paper, ${ }^{10}$ the author formulated and studied a MPD problem on a rational nodal curve. Then we may naturally expect that a solution to the MPD system on elliptic curves is approximated by a solution to the MPD system on a rational nodal curve around the boundary point. In fact, we can prove that, given

\footnotetext{
${ }^{a)}$ Electronic mail: mano@kurims.kyoto-u.ac.jp.
} 
a solution to the MPD system on a rational nodal curve, there exists a unique solution to the MPD system on elliptic curves whose "boundary value" coincides with the given solution (Theorem 3.1). The discussions developed here are similar to Ref. 3. However, we need more delicate treatment than Ref. 3 because our object is a system of partial differential equations. In Sec. IV, we construct special solutions to the MPD system on elliptic curves. Several kinds of special solutions have been found by Korotkin ${ }^{7}$ and Sasaki. ${ }^{16}$ In the present paper, we give another one. It is known that the Painlevé equations for special values of the parameters have the so-called Riccati solutions, which are linearizable. Also in our case, the MPD systems on elliptic curves with special values of parameters have linearizable solutions. We show that those solutions are written in terms of integrals of theta functions,

$$
\int_{\gamma} e^{-2 \pi \sqrt{-1} c_{0} w} \vartheta_{1}(w)^{-c_{1}} \vartheta_{1}(w-t)^{c_{1}} \mathfrak{s}\left(w-t_{i} ; \lambda\right) d w, \quad i=1,2,
$$

where we set $t_{1}=0, t_{2}=t$ and the function $\mathfrak{s}(w ; \lambda)$ is defined by

$$
\mathfrak{s}(z ; \lambda)=\frac{\vartheta_{1}(z-\lambda) \vartheta_{1}^{\prime}}{\vartheta_{1}(z) \vartheta_{1}(-\lambda)} .
$$

We call this kind of integrals Riemann-Wirtinger integrals, ${ }^{11,12,18}$ which may be regarded as an analog on elliptic curves of the hypergeometric integrals (we remark that similar integrals appear in the context of integral representations of solutions to the Knizhnik-Zamolodchikov-Bernard equation in conformal field theory on the elliptic curve $\left.{ }^{1,9}\right)$. As is proven in Theorem 3.1, the hypergeometric integrals on a rational curve asymptotically approximate the Riemann-Wirtinger integrals on elliptic curves. Applying this asymptotics, we can describe how the RiemannWirtinger integrals converge to the hypergeometric integrals when the elliptic curve degenerates to the rational curve (Theorem 4.2).

Notation for elliptic functions. In this paper, we basically follow standard notations for elliptic

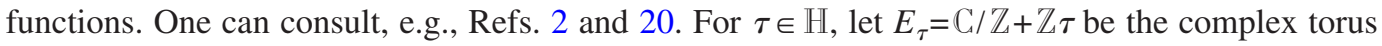
with fundamental periods 1 and $\tau$. We define Weierstrass' elliptic functions by

$$
\begin{gathered}
\zeta(z)=\frac{1}{z}+\sum^{\prime}\left(\frac{1}{z-(m+n \tau)}+\frac{1}{m+n \tau}+\frac{z}{(m+n \tau)^{2}}\right), \\
\wp(z)=-\zeta^{\prime}(z),
\end{gathered}
$$

and we introduce the function

$$
\mathfrak{z}(z ; w):=\zeta(z-w)-\zeta(z)+\zeta(w) .
$$

On the other hand, we define Jacobi's theta function by

$$
\vartheta_{1}(z)=\sqrt{-1} \sum_{n=-\infty}^{+\infty}(-1)^{n} e^{\pi \sqrt{-1}(n-1 / 2)^{2} \tau+2 \pi \sqrt{-1}(n-1 / 2) z},
$$

which is an odd function of $z$. We introduce the following functions:

$$
\begin{gathered}
\rho(z):=\vartheta_{1}^{\prime}(z) / \vartheta_{1}(z), \\
\mathfrak{s}(z ; \lambda):=\frac{\vartheta_{1}(z-\lambda) \vartheta_{1}^{\prime}}{\vartheta_{1}(z) \vartheta_{1}(-\lambda)} .
\end{gathered}
$$

The correspondence between Weierstrass and Jacobi's pictures is given by 


$$
\zeta(z)=\rho(z)+\eta_{1} z, \quad \wp(z)=-\rho^{\prime}(z)-\eta_{1},
$$

where

$$
\eta_{1}=-\frac{1}{3} \frac{\vartheta_{1}^{\prime \prime \prime}}{\vartheta_{1}^{\prime}} .
$$

\section{MPD OF LINEAR DIFFERENTIAL EQUATIONS ON ELLIPTIC CURVES}

In this section, we review the MPD theories of Fuchsian differential equations on elliptic curves. First, we follow Okamoto's formulation, which treats second-order single differential equations. Second, we follow Korotkin-Samtleben's formulation, which treats rank-two systems of linear differential equations. Thereafter, we give an explicit correspondence between these two formulations.

We consider the following second-order Fuchsian differential equation on $E_{\tau}$ :

$$
\frac{d^{2} w}{d z^{2}}=Q(z) w
$$

where

$$
Q(z)=\nu+a_{1} \wp(z)+a_{2} \wp(z-t)+\frac{3}{4} \wp\left(z-\lambda_{1}\right)+\frac{3}{4} \wp\left(z-\lambda_{2}\right)+H_{\mathfrak{z}}(z ; t)-\mu_{1} \mathfrak{z}\left(z ; \lambda_{1}\right)-\mu_{2} \mathfrak{z}\left(z ; \lambda_{2}\right) .
$$

The Riemann scheme of (1) (that is, the scheme indicating the characteristic exponents at each singular point) reads

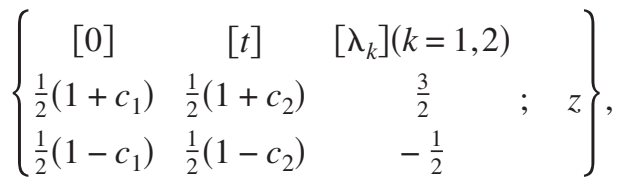

where $a_{i}=\left(c_{i}^{2}-1\right) / 4, i=1,2$.

In what follows, we make the following assumptions on the Eq. (1).

(A1) At $\lambda_{k}, k=1,2$, no solution have logarithmic singularities.

(A2) The differential equation (1) is irreducible, that is, the differential operator $\left(d^{2} / d z^{2}\right)$ $-Q(z)$ does not decompose into any product of differential operators of lower order.

(A3) Neither $c_{1}$ nor $c_{2}$ is an integer.

The coefficients $H$ and $\nu$ are expressible in terms of the other parameters by the assumption (A1),

$$
\begin{gathered}
H=M\left[\mu_{1}^{2}-\mu_{2}^{2}+N\left(\mu_{1}+\mu_{2}\right)-\Delta_{1}+\Delta_{2}\right], \\
\nu=M\left[\mu_{2}^{2} \mathfrak{z}\left(\lambda_{1} ; t\right)-\mu_{1}^{2} \mathfrak{z}\left(\lambda_{2} ; t\right)-N\left(\mu_{1} \mathfrak{z}\left(\lambda_{1} ; t\right)+\mu_{2} \mathfrak{z}\left(\lambda_{2} ; t\right)\right)+\Delta_{1} \mathfrak{z}\left(\lambda_{2} ; t\right)-\Delta_{2} \mathfrak{z}\left(\lambda_{1} ; t\right)\right],
\end{gathered}
$$

where $M=1 /\left(\mathfrak{z}\left(\lambda_{1} ; t\right)-\mathfrak{z}\left(\lambda_{2} ; t\right)\right), N=\mathfrak{z}\left(\lambda_{1} ; \lambda_{2}\right)$, and $\Delta_{k}=a_{1} \wp\left(\lambda_{k}\right)+a_{2} \wp\left(\lambda_{k}-t\right)+3 \wp\left(\lambda_{1}-\lambda_{2}\right) / 4$.

Proposition 2.1: $\left(\right.$ Okamoto $^{14}$ ) The MPD of the Fuchsian equation (1) on $E_{\tau}$ with a deformation parameter $t$ is governed by the Hamiltonian system with the Hamiltonian function $H$,

$$
\begin{gathered}
\frac{\partial \lambda_{k}}{\partial t}=\frac{\partial H}{\partial \mu_{k}}, \\
\frac{\partial \mu_{k}}{\partial t}=-\frac{\partial H}{\partial \lambda_{k}}, \quad k=1,2 .
\end{gathered}
$$


Proposition 2.2: $\left(\mathrm{Kawai}^{6}\right)$ We introduce another function by

$$
K:=\frac{1}{2 \pi \sqrt{-1}}\left[\nu+H \rho(t)-\mu_{1} \rho\left(\lambda_{1}\right)-\mu_{2} \rho\left(\lambda_{2}\right)\right],
$$

then the MPD of (1) with a deformation parameter $\tau$ is governed by the Hamiltonian system with the Hamiltonian function $K$,

$$
\begin{gathered}
\frac{\partial \lambda_{k}}{\partial \tau}=\frac{\partial K}{\partial \mu_{k}}, \\
\frac{\partial \mu_{k}}{\partial \tau}=-\frac{\partial K}{\partial \lambda_{k}}, \quad k=1,2 .
\end{gathered}
$$

Next, we consider the following system of differential equations:

$$
\frac{d Y}{d z}=A(z) Y,
$$

where

$$
A(z)=\left(\begin{array}{cc}
\alpha_{0}+\alpha_{1} \rho(z)-\alpha_{1} \rho(z-t) & \beta_{1} \mathfrak{s}(z ; \lambda)+\beta_{2} \mathfrak{s}(z-t ; \lambda) \\
\gamma_{1} \mathfrak{s}(z ;-\lambda)+\gamma_{2} \mathfrak{s}(z-t ;-\lambda) & -\alpha_{0}-\alpha_{1} \rho(z)+\alpha_{1} \rho(z-t)
\end{array}\right),
$$

with the relations

$$
-\alpha_{1}^{2}-\beta_{1} \gamma_{1}=-\frac{c_{1}^{2}}{4}, \quad-\alpha_{1}^{2}-\beta_{2} \gamma_{2}=-\frac{c_{2}^{2}}{4}
$$

for some constants $c_{1}, c_{2}$. The matrix $A(z)$ has the following quasiperiodicities:

$$
\begin{gathered}
A(z+1)=A(z) \\
A(z+\tau)=\left(\begin{array}{cc}
e^{\pi \sqrt{-1} \lambda} & 0 \\
0 & e^{-\pi \sqrt{-1} \lambda}
\end{array}\right) A(z)\left(\begin{array}{cc}
e^{-\pi \sqrt{-1} \lambda} & 0 \\
0 & e^{\pi^{\sqrt{-1} \lambda}}
\end{array}\right) .
\end{gathered}
$$

Hence, the global behavior of a fundamental system of solutions $Y(z)$ to $(8)$ is described as follows:

$$
\begin{gathered}
Y(z+1)=Y(z) M_{0}, \\
Y(z+\tau)=\left(\begin{array}{cc}
e^{\pi \sqrt{-1} \lambda} & 0 \\
0 & e^{-\pi \sqrt{-1} \lambda}
\end{array}\right) Y(z) M_{\infty}
\end{gathered}
$$

and

$$
\begin{gathered}
Y(z)=G_{1}(I+O(z))\left(\begin{array}{cc}
z^{c_{1} / 2} & 0 \\
0 & z^{-c_{1} / 2}
\end{array}\right) C_{1} \quad \text { at } z=0, \\
=G_{2}(I+O(z-t))\left(\begin{array}{cc}
(z-t)^{c_{2} / 2} & 0 \\
0 & (z-t)^{-c_{2} / 2}
\end{array}\right) C_{2} \quad \text { at } z=t,
\end{gathered}
$$

where $M_{0}, M_{\infty}, C_{1}, C_{2} \in S L(2, \mathrm{C})$.

Definition 2.1: We put 


$$
\begin{aligned}
& M_{1}=C_{1}^{-1}\left(\begin{array}{cc}
e^{\pi \sqrt{ }-1} c_{1} & 0 \\
0 & e^{-\pi \sqrt{-1} c_{1}}
\end{array}\right) C_{1}, \\
& M_{2}=C_{2}^{-1}\left(\begin{array}{cc}
e^{\pi \sqrt{ }-1} c_{2} & 0 \\
0 & e^{-\pi \sqrt{-1} c_{2}}
\end{array}\right) C_{2} .
\end{aligned}
$$

For a fundamental system of solutions $Y(z)$ to (8), we define the monodromy data associated with $Y(z)$ by the set of matrices $\left\{M_{0}, M_{\infty}, M_{1}, M_{2}\right\}$. We note that these matrices obey the unique relation

$$
M_{\infty}^{-1} M_{0}^{-1} M_{\infty} M_{0}=M_{1} M_{2} .
$$

Proposition 2.3: (Korotkin-Samtleben ${ }^{8}$ ) Let $Y(z ; t)$ be a family of fundamental systems of solutions to (8). Then the monodromy matrices of $Y(z ; t)$ are independent of $t$, if and only if $Y(z ; t)$ and $\lambda=\lambda(t)$ satisfy the following system of differential equations:

$$
\frac{\partial Y}{\partial t}(z ; t)=B(z ; t) Y(z ; t), \quad \frac{\partial \lambda}{\partial t}=-2 \alpha_{1},
$$

where

$$
B(z ; t)=\left(\begin{array}{cc}
\varepsilon+\alpha_{1} \rho(z-t) & -\beta_{2} \mathfrak{s}(z-t ; \lambda) \\
-\gamma_{2} \mathfrak{s}(z-t ;-\lambda) & -\varepsilon-\alpha_{1} \rho(z-t)
\end{array}\right)
$$

and $\varepsilon$ is some function of $t$ and independent of $z$.

Remark 2.1: The parameter $\varepsilon$ is not essential because it comes from ambiguity of the normalization of $Y(z ; t)$.

Proposition 2.4: (Reference 8) Let $Y(z ; \tau)$ be a family of fundamental systems of solutions to (8). Then the monodromy matrices of $Y(z ; \tau)$ are independent of $\tau$, if and only if $Y(z ; \tau)$ and $\lambda$ $=\lambda(\tau)$ satisfy the following system of differential equations:

$$
\frac{\partial Y}{\partial \tau}(z ; \tau)=C(z ; \tau) Y(z ; \tau), \quad \pi \sqrt{-1} \frac{\partial \lambda}{\partial \tau}=\alpha_{0}
$$

where

$$
\quad=\left(\begin{array}{cc}
\delta+\frac{\alpha_{1}}{4 \pi \sqrt{-1}}\left(\rho(z)^{2}+\rho^{\prime}(z)-\rho(z-t)^{2}-\rho^{\prime}(z-t)\right) & -\frac{\beta_{1}}{2 \pi \sqrt{-1}} \frac{\partial \mathfrak{s}}{\partial \lambda}(z ; \lambda)-\frac{\beta_{2}}{2 \pi \sqrt{-1}} \frac{\partial \mathfrak{s}}{\partial \lambda}(z-t ; \lambda) \\
-\frac{\gamma_{1}}{2 \pi \sqrt{-1}} \frac{\partial \mathfrak{s}}{\partial \lambda}(z ;-\lambda)-\frac{\gamma_{2}}{2 \pi \sqrt{-1}} \frac{\partial \mathfrak{s}}{\partial \lambda}(z-t ;-\lambda) & -\delta-\frac{\alpha_{1}}{4 \pi \sqrt{-1}}\left(\rho(z)^{2}+\rho^{\prime}(z)-\rho(z-t)^{2}-\rho^{\prime}(z-t)\right)
\end{array}\right)
$$

and $\delta$ is some function of $\tau$ and independent of $z$.

In this paper, we choose $\varepsilon, \delta$ such as $\varepsilon=-\pi \sqrt{-1} \alpha_{1}, \delta=\left(\alpha_{0}-\pi \sqrt{-1} c_{0}\right) / 2$, respectively, for some constant $c_{0}$. From the integrability conditions between (8), (16), and (18),

$$
\frac{\partial A}{\partial t}-\frac{\partial B}{\partial z}+[A, B]=0, \quad \frac{\partial A}{\partial \tau}-\frac{\partial C}{\partial z}+[A, C]=0,
$$

we obtain the following MPD system of differential equations for the coefficients of $A(z):^{8}$

$$
\frac{\partial \lambda}{\partial t}=-2 \alpha_{1},
$$




$$
\begin{gathered}
\frac{\partial \alpha_{0}}{\partial t}=-\beta_{1} \gamma_{2} \frac{\partial \mathfrak{s}}{\partial \lambda}(t ; \lambda)+\beta_{2} \gamma_{1} \frac{\partial \mathfrak{s}}{\partial \lambda}(t ;-\lambda), \\
\frac{\partial \alpha_{1}}{\partial t}=-\beta_{1} \gamma_{2} \mathfrak{s}(t ; \lambda)+\beta_{2} \gamma_{1} \mathfrak{s}(t ;-\lambda), \\
\frac{\partial \beta_{1}}{\partial t}=-2 \pi \sqrt{-1} \alpha_{1} \beta_{1}-2 \alpha_{1} \beta_{1} \rho(t)-2 \alpha_{1} \beta_{2} \mathfrak{s}(t ;-\lambda), \\
\frac{\partial \beta_{2}}{\partial t}=-2 \pi \sqrt{-1} \alpha_{1} \beta_{2}+2 \alpha_{0} \beta_{2}+2 \alpha_{1} \beta_{2} \rho(t)+2 \alpha_{1} \beta_{1} \mathfrak{s}(t ; \lambda), \\
\frac{\partial \gamma_{1}}{\partial t}=2 \pi \sqrt{-1} \alpha_{1} \gamma_{1}+2 \alpha_{1} \gamma_{1} \rho(t)+2 \alpha_{1} \gamma_{2} \mathfrak{s}(t ; \lambda), \\
\frac{\partial \gamma_{2}}{\partial t}=2 \pi \sqrt{-1} \alpha_{1} \gamma_{2}-2 \alpha_{0} \gamma_{2}-2 \alpha_{1} \gamma_{2} \rho(t)-2 \alpha_{1} \gamma_{1} \mathfrak{s}(t ;-\lambda),
\end{gathered}
$$

and

$$
\begin{gathered}
2 \pi \sqrt{-1} \frac{\partial \lambda}{\partial \tau}=2 \alpha_{0} \\
2 \pi \sqrt{-1} \frac{\partial \alpha_{0}}{\partial \tau}=-\left(\beta_{1} \gamma_{1}+\beta_{2} \gamma_{2}\right) \rho^{\prime \prime}(\lambda)+\beta_{1} \gamma_{2} \frac{\partial^{2} \mathfrak{s}}{\partial \lambda^{2}}(t ; \lambda)-\beta_{2} \gamma_{1} \frac{\partial^{2} \mathfrak{s}}{\partial \lambda^{2}}(t ;-\lambda) \\
2 \pi \sqrt{-1} \frac{\partial \alpha_{1}}{\partial \tau}=\beta_{1} \gamma_{2} \frac{\partial \mathfrak{s}}{\partial \lambda}(t ; \lambda)-\beta_{2} \gamma_{1} \frac{\partial \mathfrak{s}}{\partial \lambda}(t ;-\lambda) \\
2 \pi \sqrt{-1} \frac{\partial \beta_{1}}{\partial \tau}=2 \pi \sqrt{-1}\left(\alpha_{0}-\pi \sqrt{-1} c_{0}\right) \beta_{1}+2 \alpha_{1} \beta_{2} \frac{\partial \mathfrak{s}}{\partial \lambda}(t ;-\lambda)+\alpha_{1} \beta_{1}\left(2 \wp(\lambda)-\rho(t)^{2}+\wp(t)\right) \\
2 \pi \sqrt{-1} \frac{\partial \beta_{2}}{\partial \tau}=2 \pi \sqrt{-1}\left(\alpha_{0}-\pi \sqrt{-1} c_{0}\right) \beta_{2}-2 \alpha_{1} \beta_{1} \frac{\partial \mathfrak{s}}{\partial \lambda}(t ; \lambda)-\alpha_{1} \beta_{2}\left(2 \wp(\lambda)-\rho(t)^{2}+\wp(t)\right) \\
2 \pi \sqrt{-1} \frac{\partial \gamma_{1}}{\partial \tau}=-2 \pi \sqrt{-1}\left(\alpha_{0}-\pi \sqrt{-1} c_{0}\right) \gamma_{1}-2 \alpha_{1} \gamma_{2} \frac{\partial \mathfrak{s}}{\partial \lambda}(t ; \lambda)-\alpha_{1} \gamma_{1}\left(2 \wp(\lambda)-\rho(t)^{2}+\wp(t)\right) \\
\frac{\partial \gamma}{2}=-2 \pi \sqrt{-1}\left(\alpha_{0}-\pi \sqrt{-1} c_{0}\right) \gamma_{2}+2 \alpha_{1} \gamma_{1} \frac{\partial \mathfrak{s}}{\partial \lambda}(t ;-\lambda)+\alpha_{1} \gamma_{2}\left(2 \wp(\lambda)-\rho(t)^{2}+\wp(t)\right)
\end{gathered}
$$


Remark 2.2: This system of differential equations (20)-(33) is left invariant by the following transformation:

$$
\left(\lambda, \alpha_{0}, \alpha_{1}, \beta_{1}, \beta_{2}, \gamma_{1}, \gamma_{2}, \varepsilon, \delta\right) \mapsto\left(-\lambda,-\alpha_{0},-\alpha_{1}, \gamma_{1}, \gamma_{2}, \beta_{1}, \beta_{2},-\varepsilon,-\delta\right) .
$$

Proposition 2.5: Two systems of the MPD equations (5), (7), and (20)-(33) are equivalent to each other. In particular, the explicit correspondence between the dependent variables of two systems is given as follows:

$$
\begin{gathered}
\lambda_{1}+\lambda_{2}=\lambda+t, \\
\beta_{1}+\beta_{2} \mathfrak{s}(t ; \lambda)^{-1}\left(\rho\left(\lambda-\lambda_{1}\right)+\rho\left(\lambda-\lambda_{2}\right)+\rho(t)-\rho(\lambda)\right)=0, \\
\mu_{1}=\alpha_{0}-\left(\alpha_{1}-1 / 2\right) \rho\left(\lambda_{1}-t\right)+\left(\alpha_{1}+1 / 2\right) \rho\left(\lambda_{1}\right)-\frac{1}{2} \rho\left(\lambda_{1}-\lambda_{2}\right), \\
\mu_{2}=\alpha_{0}-\left(\alpha_{1}-1 / 2\right) \rho\left(\lambda_{2}-t\right)+\left(\alpha_{1}+1 / 2\right) \rho\left(\lambda_{2}\right)-\frac{1}{2} \rho\left(\lambda_{2}-\lambda_{1}\right) .
\end{gathered}
$$

Note that $\gamma_{1}$ and $\gamma_{2}$ can be recovered from the relations in (10).

Proof: First of all, note that the function

$$
\beta_{1}+\beta_{2} \mathfrak{s}(z ; \lambda)^{-1} \mathfrak{s}(z-t ; \lambda)
$$

is meromorphic in $z$ and has two zeros on $E_{\tau}$, which we denote by $\left[\lambda_{1}\right],\left[\lambda_{2}\right]$. Here we use the symbol $[\cdot]$ in order to indicate an equivalence class modulo the period lattice. We can choose complex numbers $\lambda_{1}, \lambda_{2}$ which are representatives for $\left[\lambda_{1}\right],\left[\lambda_{2}\right]$, respectively, such as $\lambda_{1}+\lambda_{2}=\lambda$ $+t$ by Abel's theorem. We introduce the following function of $z$ :

$$
r(z)=\mathfrak{s}\left(z ; \lambda_{1}\right) \mathfrak{s}\left(z-t ; \lambda-\lambda_{1}\right) \mathfrak{s}\left(t ; \lambda-\lambda_{1}\right) .
$$

Then $r(z)$ is a quasiperiodic function whose quasiperiodicity is given by

$$
r(z+1)=r(z), \quad r(z+\tau)=e^{2 \pi \sqrt{-1} \lambda} r(z),
$$

and has poles $[0],[t]$ and zeros $\left[\lambda_{1}\right],\left[\lambda_{2}\right]$. We transform the dependent variables of the system $(8)$ into $W(z)=R(z) Y(z)$, where

$$
R(z)=\left(\begin{array}{cc}
r(z)^{-1 / 2} & 0 \\
0 & r(z)^{1 / 2}
\end{array}\right)
$$

If we find the single differential equation for the first component of $W(z)=\left(\begin{array}{l}w_{1}(z) \\ w_{2}(z)\end{array}\right)$, then we obtain a differential equation of the type (1), which has two apparent singularities $\left[\lambda_{1}\right],\left[\lambda_{2}\right]$. By computing the coefficients of that equation, we obtain the relations (34)-(37).

\section{ASYMPTOTIC ANALYSIS ON SOLUTIONS TO THE MPD SYSTEM AROUND $q=0$}

In this section, we study asymptotic behaviors of solutions to the MPD system in Sec. II. In Ref. 10 the author formulated and solved the MPD system on a rational nodal curve with two regular singular points. Then we may expect that solutions to the MPD system on elliptic curves are approximated by solutions to the MPD system on the rational nodal curve around the boundary point in the moduli space of elliptic curves. We shall prove that it is valid under some condition (Theorem 3.1).

First of all, we recall the standard MPD theory on $\mathrm{P}^{1}(\mathrm{C})$ with four regular singular points $\{0,1, s, \infty\}$ : consider 


$$
\frac{d Z}{d x}=B(x) Z,
$$

where

$$
\begin{gathered}
B(x)=\frac{B_{0}}{x}+\frac{B_{1}}{x-1}+\frac{B_{2}}{x-s}, \\
B_{i}=\left(\begin{array}{cc}
p_{i} & q_{i} \\
r_{i} & -p_{i}
\end{array}\right), \quad i=0,1,2,
\end{gathered}
$$

and assume that

$$
\begin{gathered}
B_{\infty}:=-B_{0}-B_{1}-B_{2}=\left(\begin{array}{cc}
-c_{0} / 2 & 0 \\
0 & c_{0} / 2
\end{array}\right), \\
-p_{i}^{2}-q_{i} r_{i}=-\frac{c_{i}^{2}}{4}, \quad i=0,1,2,
\end{gathered}
$$

for some constants $c_{0}, c_{1}, c_{2}$. We take a fundamental system of solutions normalized as follows:

$$
\begin{aligned}
Z(x) & =\left(I+O\left(x^{-1}\right)\right) x^{T_{0}} \quad \text { at } x=\infty \\
& =K_{1}(I+O(x-1))(x-1)^{T_{1}} C_{1} \quad \text { at } x=1 \\
& =K_{2}(I+O(x-s))(x-s)^{T_{2}} C_{2} \quad \text { at } x=s \\
& =K_{0}(I+O(x)) x^{T_{0}} C_{0} \quad \text { at } x=0,
\end{aligned}
$$

where $T_{i}=\left(\begin{array}{cc}c_{i} / 2 & 0 \\ 0 & -c_{i} / 2\end{array}\right), i=0,1,2$. We define the monodromy data associated with $Z(x)$ by the following set of matrices:

$$
\left\{N_{\infty}=e^{-2 \pi \sqrt{-1} T_{0}}, \quad N_{i}=C_{i}^{-1} e^{2 \pi \sqrt{-1} T_{i}} C_{i}\right\}_{i=0,1,2}
$$

We note that these matrices obey the unique relation

$$
N_{\infty} N_{0} N_{1} N_{2}=I
$$

The following fact is well known.

Proposition 3.1: (Jimbo-Miwa-Ueno ${ }^{4}$ ) Let $Z(x ; s)$ be a family of fundamental solutions to (40). Then the monodromy data associated with $Z(x ; s)$ are independent of $s$, if and only if the coefficients $\left\{B_{i}\right\}_{i=0,1,2}$ satisfy the following system of differential equations, which is called the Schlesinger system:

$$
\begin{gathered}
\frac{d B_{0}}{d s}=\frac{1}{s}\left[B_{2}, B_{0}\right], \\
\frac{d B_{1}}{d s}=\frac{1}{s-1}\left[B_{2}, B_{1}\right], \\
\frac{d B_{2}}{d s}=-\frac{1}{s}\left[B_{2}, B_{0}\right]-\frac{1}{s-1}\left[B_{2}, B_{1}\right] .
\end{gathered}
$$

Moreover, if the connection matrix $C_{0}$ is also independent of $s$, then $K_{0}$ in (44) satisfies the following differential equation: 


$$
\frac{d K_{0}}{d s}=\Theta_{0} K_{0}
$$

where $\Theta_{0}=(1 / s) B_{2}$.

We introduce the $\tau$-function associated with a solution $\left\{B_{0}, B_{1}, B_{2}\right\}$ to (47) by

$$
\frac{d}{d s} \log \tau(s)=\operatorname{tr}\left(\frac{B_{0}}{s}+\frac{B_{1}}{s-1}\right) B_{2} .
$$

Proposition 3.2: (Jimbo-Miwa $\left.{ }^{5}\right)$ The components $\left(K_{0}\right)_{a b}(a, b \in\{1,2\})$ of the solution matrix $K_{0}$ to (48) can be written in terms of $\tau$-quotients associated with $\left\{B_{0}, B_{1}, B_{2}\right\}$,

$$
\left(K_{0}\right)_{a b}=\text { const } q\left\{\begin{array}{ll}
\infty & 0 \\
a & b
\end{array} ; \quad B_{0}, B_{1}, B_{2}\right\},
$$

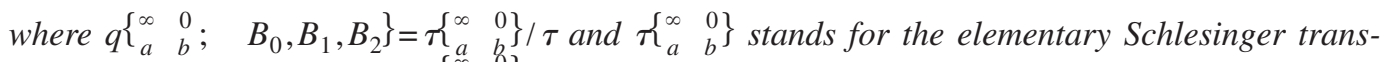

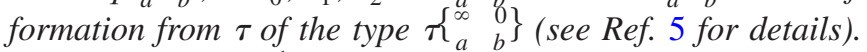

Noting that $K_{0}^{-1} B_{0} K_{0}=T_{0}$ and det $K_{0}=1$, we have the following expression of $K_{0}$ :

$$
K_{0}=\left(\begin{array}{cc}
k & -q_{0}\left(c_{0} k\right)^{-1} \\
-q_{0}^{-1}\left(p_{0}-\frac{c_{0}}{2}\right) k & \left(c_{0} k\right)^{-1}\left(p_{0}+\frac{c_{0}}{2}\right)
\end{array}\right)
$$

and

$$
k=\text { const } q\left\{\begin{array}{ll}
\infty & 0 \\
1 & 1
\end{array} ; \quad B_{0}, B_{1}, B_{2}\right\} .
$$

Remark 3.1: It was proved in Ref. 10 that $\left\{B_{i}\right\}_{i=0,1,2}$ and $k$ solve the MPD system on a rational nodal curve with two regular singular points.

In what follows, we use the notations $q=e^{2 \pi \sqrt{-1}}, x=e^{2 \pi \sqrt{-1}} z, \xi=e^{2 \pi \sqrt{-1} \lambda}, s=e^{2 \pi \sqrt{-1}}$. Before we proceed to the asymptotic analysis on the MPD system (20)-(33), we explain a basic idea about it. Suppose that there exists a solution $\left\{\lambda, \alpha_{0}, \alpha_{1}, \beta_{1}, \beta_{2}, \gamma_{1}, \gamma_{2}\right\}$ to the MPD system (20)-(33), such that they converge as follows:

$$
\begin{gathered}
\xi=e^{2 \pi \sqrt{-1} \lambda} \rightarrow 0, \\
\alpha_{i} \rightarrow \alpha_{i}^{0}, \quad i=0,1, \\
\beta_{i} \rightarrow \beta_{i}^{0}, \quad i=1,2, \\
\gamma_{i} \rightarrow \gamma_{i}^{0}, \quad i=1,2,
\end{gathered}
$$

as $q \rightarrow 0$, where $\alpha_{i}^{0}=\alpha_{i}^{0}(s), \beta_{i}^{0}=\beta_{i}^{0}(s), \gamma_{i}^{0}=\gamma_{i}^{0}(s)$ are meromorphic functions of $s$. Then the associated linear differential equation (8) should converge to

$$
\frac{d}{d x} Y_{0}(x)=A^{0}(x) Y_{0}(x)
$$

where

$$
A^{0}(x)=\frac{A_{0}^{0}}{x}+\frac{A_{1}^{0}}{x-1}+\frac{A_{2}^{0}}{x-s}
$$

and 


$$
\begin{gathered}
A_{0}^{0}=\left(\begin{array}{cc}
\alpha_{0}^{0} / 2 \pi \sqrt{-1} & 0 \\
-\left(\gamma_{1}^{0}+\gamma_{2}^{0}\right) & -\alpha_{0}^{0} / 2 \pi \sqrt{-1}
\end{array}\right), \\
A_{1}^{0}=\left(\begin{array}{cc}
\alpha_{1}^{0} & \beta_{1}^{0} \\
\gamma_{1}^{0} & -\alpha_{1}^{0}
\end{array}\right), \quad A_{2}^{0}=\left(\begin{array}{cc}
-\alpha_{1}^{0} & \beta_{2}^{0} \\
\gamma_{2}^{0} & \alpha_{1}^{0}
\end{array}\right) .
\end{gathered}
$$

The differential equation (51) is related to (40) by the gauge transformation,

$$
B_{i}=G^{-1} A_{i}^{0} G, \quad i=0,1,2,
$$

with

$$
G=\left(\begin{array}{cc}
1 & -\frac{\pi \sqrt{-1}\left(\beta_{1}^{0}+\beta_{2}^{0}\right)}{\alpha_{0}^{0}} \\
0 & 1
\end{array}\right) .
$$

Since the monodromy data should be preserved along the solution of the MPD system, we see that the monodromy data $\left\{M_{0}, M_{\infty}, M_{1}, M_{2}\right\}$ on the elliptic curve should correspond to the monodromy data $\left\{N_{0}, N_{\infty}, N_{1}, N_{2}\right\}$ on the rational curve as follows:

$$
\begin{gathered}
N_{\infty}=M_{0}^{-1}, \quad N_{1}=M_{1}, \quad N_{2}=M_{2}, \\
N_{0}=\left(N_{1} N_{2} N_{\infty}\right)^{-1}=M_{\infty}^{-1} M_{0} M_{\infty} .
\end{gathered}
$$

However, this correspondence between two monodromy data is not one to one. So we give attention to the differential equation (27). We could expect that $\xi$ behaves like $\xi \sim q^{c_{0}} \xi_{0}$ as $q$ $\rightarrow 0$ by noting that $\alpha_{0}^{0}=\pi \sqrt{-1} c_{0}$. Substituting it into (12), we have

$$
\left(\begin{array}{cc}
q^{-c_{0} / 2} & 0 \\
0 & q^{c_{0} / 2}
\end{array}\right) Y(q x ; q) \rightarrow\left(\begin{array}{cc}
\xi_{0}^{1 / 2} & 0 \\
0 & \xi_{0}^{-1 / 2}
\end{array}\right) Y_{0}(x) M_{\infty},
$$

as $q \rightarrow 0$, which suggests that the monodromy matrix $M_{\infty}$ corresponds to the connection matrix $C_{0}$ in (44) joining $x=\infty$ and $x=0$ on the rational curve. Actually, put

$$
M_{\infty}=C_{0}, \quad M_{0}=C_{0} N_{0} C_{0}^{-1}, \quad M_{1}=N_{1}, \quad M_{2}=N_{2},
$$

then we see that they must obey the relation

$$
M_{\infty}^{-1} M_{0}^{-1} M_{\infty} M_{0}=M_{1} M_{2}
$$

because we have $M_{\infty}^{-1} M_{0}^{-1} M_{\infty} M_{0}=N_{0}^{-1} N_{\infty}^{-1}=N_{1} N_{2}$. The relation (53) coincides with the one among monodromy matrices on the elliptic curve (15). Therefore, the correspondence (52) between the data $\left\{N_{\infty}, N_{0}, N_{1}, N_{2}, C_{0}\right\}$ and $\left\{M_{\infty}, M_{0}, M_{1}, M_{2}\right\}$ is one to one. By the above consideration and Proposition 3.1 and 3.2, we may expect that a set of solutions $\left\{B_{0}, B_{1}, B_{2}, k\right\}$ to the Schlesinger system (47) and the differential equation (48) uniquely corresponds to a solution $\left\{\lambda, \alpha_{0}, \alpha_{1}, \beta_{1}, \beta_{2}, \gamma_{1}, \gamma_{2}\right\}$ to (20)-(33) in the limit of $q \rightarrow 0$.

Theorem 3.1: We assume $0<\Re c_{0}<1$ and choose a constant $\delta$ satisfying $0<\delta$ $<\min \left(\Re c_{0}, 1-\Re c_{0}\right)$. For a given set of solutions $\left\{B_{0}, B_{1}, B_{2}, k\right\}$ to the Schlesinger system (47) and the differential equation (48), put

$$
\begin{gathered}
\xi_{0}=\left(\frac{c_{0} k}{p_{0}+c_{0} / 2}\right)^{2}, \\
\alpha_{0}^{0}=\pi \sqrt{-1} c_{0},
\end{gathered}
$$




$$
\begin{gathered}
\alpha_{1}^{0}=-p_{2}-\frac{q_{0} r_{2}}{p_{0}+c_{0} / 2}, \\
\beta_{i}^{0}=q_{i}-\frac{2 q_{0} p_{i}}{p_{0}+c_{0} / 2}-\left(\frac{q_{0}}{p_{0}+c_{0} / 2}\right)^{2} r_{i}, \quad i=1,2, \\
\gamma_{i}^{0}=r_{i}, \quad i=1,2 .
\end{gathered}
$$

We introduce the following subset in $\mathrm{P}^{1}(\mathrm{C})$ :

$$
\begin{aligned}
E_{\left\{B_{i}\right\}, k}= & \left\{\text { poles of the functions } B_{i}(i=0,1,2) \text { and } k\right\} \cup\left\{\text { zeros of the functions } p_{0}\right. \\
& \left.+c_{0} / 2 \text { and } k\right\}
\end{aligned}
$$

and take a simply connected relatively compact open set $V \subset \mathbb{P}^{1}(C) \backslash\left(\{0,1, \infty\} \cup E_{\left\{B_{i}\right\}, k}\right)$, then there exists a constant $K>0$, such that $1 / K<\left|\xi_{0}(s)\right|<K,\left|\alpha_{j}^{0}(s)\right|<K,\left|\beta_{i}^{0}(s)\right|<K,\left|\gamma_{i}^{0}(s)\right|<K \quad(j$ $=0,1, \quad i=1,2)$ for any $s \in V$. Then, for any $\varphi>0$, there exists an $\varepsilon>0$, such that (20)-(33) admit a unique solution $\left\{\xi, \alpha_{0}, \alpha_{1}, \beta_{1}, \beta_{2}, \gamma_{1}, \gamma_{2}\right\}$ in the sector $S_{\varepsilon, \varphi}=\{q \in \mathrm{C}|0<| q|<\varepsilon,| \arg q \mid<\varphi\}$ with the properties

$$
\begin{gathered}
\left|q^{-c_{0}} \xi-\xi_{0}\right| \leq K|q|^{\delta}, \quad\left|\alpha_{0}-\alpha_{0}^{0}\right| \leq L|q|^{\delta}, \quad\left|\alpha_{1}-\alpha_{1}^{0}\right| \leq K|q|^{\delta}, \\
\left|\beta_{i}-\beta_{i}^{0}\right| \leq K|q|^{\delta}, \quad\left|\gamma_{i}-\gamma_{i}^{0}\right| \leq K|q|^{\delta}, \quad i=1,2,
\end{gathered}
$$

where $L$ is a constant such as $0<L<\pi \delta / 2$. These estimates are uniform on $s \in V$.

Proof: Since the proof is quite technical, we give only an outline. The discussion here is very similar to Refs. 17 and 3. We construct a solution of (27)-(33) with respect to the variable $q$ by successive approximation starting from $\left\{\xi_{0}, \alpha_{0}^{0}, \alpha_{1}^{0}, \beta_{1}^{0}, \beta_{2}^{0}, \gamma_{1}^{0}, \gamma_{2}^{0}\right\}$ : we define $\xi^{(k)}(q), \alpha_{0}^{(k)}(q), \alpha_{1}^{(k)}$ $\times(q), \beta_{i}^{(k)}(q), \gamma_{i}^{(k)}(q)(k=0,1,2, \ldots)$ inductively by $\xi^{(0)}(q)=\xi_{0}, \ldots, \quad \gamma_{2}^{(0)}(q)=\gamma_{2}^{0}$ and

$$
\begin{gathered}
q^{-c_{0}} \xi^{(k)}(q)=\xi_{0}+\int_{0}^{q} \frac{\alpha_{0}^{(k-1)}\left(q^{\prime}\right)-\pi \sqrt{-1} c_{0}}{\pi \sqrt{-1} q^{\prime}} q^{\prime-c_{0}} \xi^{(k-1)}\left(q^{\prime}\right) d q^{\prime}, \\
\alpha_{0}^{(k)}(q)=\alpha_{0}^{0}+\int_{0}^{q} \frac{R_{\alpha_{0}}\left(\xi^{(k-1)}\left(q^{\prime}\right), \alpha_{i}^{(k-1)}\left(q^{\prime}\right), \beta_{i}^{(k-1)}\left(q^{\prime}\right), \gamma_{i}^{(k-1)}\left(q^{\prime}\right) ; s, q^{\prime}\right)}{(2 \pi \sqrt{-1})^{2} q^{\prime}} d q^{\prime}, \\
\alpha_{1}^{(k)}(q)=\alpha_{1}^{0}+\int_{0}^{q} \frac{R_{\alpha_{1}}\left(\xi^{(k-1)}\left(q^{\prime}\right), \alpha_{0}^{(k-1)}\left(q^{\prime}\right), \ldots, q^{\prime}\right)}{(2 \pi \sqrt{-1})^{2} q^{\prime}} d q^{\prime}, \\
\beta_{i}^{(k)}(q)=\beta_{i}^{0}+\int_{0}^{q} \frac{R_{\beta_{i}}\left(\xi^{(k-1)}\left(q^{\prime}\right), \alpha_{0}^{(k-1)}\left(q^{\prime}\right), \ldots, q^{\prime}\right)}{(2 \pi \sqrt{-1})^{2} q^{\prime}} d q^{\prime}, \quad i=1,2, \\
\gamma_{i}^{(k)}(q)=\gamma_{i}^{0}+\int_{0}^{q} \frac{R_{\gamma_{i}}\left(\xi^{(k-1)}\left(q^{\prime}\right), \alpha_{0}^{(k-1)}\left(q^{\prime}\right), \ldots, q^{\prime}\right)}{(2 \pi \sqrt{-1})^{2} q^{\prime}} d q^{\prime}, \quad i=1,2,
\end{gathered}
$$

where $R_{\alpha_{0}}\left(\xi, \alpha_{i}, \beta_{i}, \gamma_{i} ; s, q\right), \ldots, R_{\gamma_{2}}\left(\xi, \alpha_{i}, \beta_{i}, \gamma_{i} ; s, q\right)$ denote the right hand sides of the Eqs. (28)-(33) and the path of integrals is the line segment $\left\{q^{\prime}=r e^{i \theta}|0<r<| q \mid, \quad \theta=\arg q\right\}$. Then we can prove that $\left\{\xi=\lim _{k \rightarrow \infty} \xi^{(k)}, \ldots, \quad \gamma_{2}=\lim _{k \rightarrow \infty} \gamma_{2}^{(k)}\right\}$ gives a unique solution to (27)-(33) satisfying the inequalities (54) and (55). Next, we have to verify that the solution $\left\{\xi, \alpha_{i}, \beta_{i}, \gamma_{i}\right\}$ con- 
structed in above satisfies the differential equations (20)-(26) with respect to the variable $s$. We consider the following differential equation:

$$
\frac{\partial Y(x)}{\partial x}=A^{0}(x) Y(x),
$$

where

$$
A^{0}(x)=\frac{A_{0}^{0}}{x}+\frac{A_{1}^{0}}{x-1}+\frac{A_{2}^{0}}{x-s}
$$

and

$$
\begin{gathered}
A_{0}^{0}=\left(\begin{array}{cc}
\alpha_{0}^{0} / 2 \pi \sqrt{-1} & 0 \\
-\left(\gamma_{1}^{0}+\gamma_{2}^{0}\right) & -\alpha_{0}^{0} / 2 \pi \sqrt{-1}
\end{array}\right), \\
A_{1}^{0}=\left(\begin{array}{cc}
\alpha_{1}^{0} & \beta_{1}^{0} \\
\gamma_{1}^{0} & -\alpha_{1}^{0}
\end{array}\right), \quad A_{2}^{0}=\left(\begin{array}{cc}
-\alpha_{1}^{0} & \beta_{2}^{0} \\
\gamma_{2}^{0} & \alpha_{1}^{0}
\end{array}\right) .
\end{gathered}
$$

Let $Y_{0}(x)$ be a fundamental solution to (56) normalized as follows:

$$
\begin{aligned}
& Y_{0}(x)=G\left(I+O\left(x^{-1}\right)\right) x^{T_{0}} \quad \text { at } x=\infty \\
= & G_{1}^{0}(I+O(x-1))(x-1)^{T_{1}} C_{1} \quad \text { at } x=1 \\
= & G_{2}^{0}(I+O(x-s))(x-s)^{T_{2}} C_{2} \quad \text { at } x=s \\
= & G_{0}^{0}(I+O(x)) x^{T_{0}} C_{0} \quad \text { at } x=0,
\end{aligned}
$$

where $G=\left(\begin{array}{cc}1 & q_{0}\left(p_{0}+c_{0} / 2\right)^{-1} \\ 0 & 1\end{array}\right)$ and $G_{0}^{0}$ is defined by $G_{0}^{0}=\left(\begin{array}{cc}l & 0 \\ r_{0} l / c_{0} & l^{-1}\end{array}\right)$ with some $l \neq 0$. Put

$$
\begin{gathered}
F(z ; q)=\frac{1}{2 \pi \sqrt{-1} q}\left(\begin{array}{c}
\frac{\alpha_{0}-\pi \sqrt{-1} c_{0}}{2}+\frac{\alpha_{1}}{4 \pi \sqrt{-1}}\left(\rho(z)^{2}+\rho^{\prime}(z)-\rho(z-t)^{2}-\rho^{\prime}(z-t)\right) \\
-\frac{\gamma_{1}}{2 \pi \sqrt{-1}} \frac{\partial \mathfrak{s}}{\partial \lambda}(z ;-\lambda)-\frac{\gamma_{2}}{2 \pi \sqrt{-1}} \frac{\partial \mathfrak{s}}{\partial \lambda}(z-t ;-\lambda) \\
-\frac{\beta_{1}}{2 \pi \sqrt{-1}} \frac{\partial \mathfrak{s}}{\partial \lambda}(z ; \lambda)-\frac{\beta_{2}}{2 \pi \sqrt{-1}} \frac{\partial \mathfrak{s}}{\partial \lambda}(z-t ; \lambda) \\
-\frac{\alpha_{0}-\pi \sqrt{-1} c_{0}}{2}-\frac{\alpha_{1}}{4 \pi \sqrt{-1}}\left(\rho(z)^{2}+\rho^{\prime}(z)-\rho(z-t)^{2}-\rho^{\prime}(z-t)\right)
\end{array}\right),
\end{gathered}
$$

and we set

$$
U(z ; q)=1+\sum_{k=1}^{\infty} \int_{0}^{q} d q_{1} \int_{0}^{q_{1}} d q_{2} \cdots \int_{0}^{q_{k-1}} d q_{k} F\left(z ; q_{1}\right) F\left(z ; q_{2}\right) \cdots F\left(z ; q_{k}\right) .
$$

The integration is taken along the line segment joining 0 and $q$ in $S_{\varepsilon, \varphi}$. Then we can prove that the infinite sum in the right hand side is uniformly convergent on $s \in V$. Put

$$
Y(z ; q)=U(z ; q) Y_{0}(x),
$$

then it satisfies the linear differential equation (8) associated with $\left\{\xi, \alpha_{i}, \beta_{i}, \gamma_{i}\right\}$ and $\lim _{q \rightarrow 0} Y(z ; q)=Y_{0}(x)$. By construction, it is obvious that the monodromy matrices $M_{0}, M_{1}, M_{2}$ are 
independent of $s$. Hence it is enough to prove that the matrix $M_{\infty}$ is also independent of $s$. By (12), we have

$$
\lim _{q \rightarrow 0}\left(\begin{array}{cc}
q^{-c_{0} / 2} & 0 \\
0 & q^{c_{0} / 2}
\end{array}\right) Y(q x ; q)=\left(\begin{array}{cc}
\xi_{0}^{1 / 2} & 0 \\
0 & \xi_{0}^{-1 / 2}
\end{array}\right) Y_{0}(x) M_{\infty} .
$$

On the other hand, making use of the expansion of $Y_{0}(x)$ around $x=0$, we have

$$
\left(\begin{array}{cc}
q^{-c_{0} / 2} & 0 \\
0 & q^{c_{0} / 2}
\end{array}\right) Y_{0}(q x)=\left(\begin{array}{cc}
q^{-c_{0} / 2} & 0 \\
0 & q^{c_{0} / 2}
\end{array}\right) G_{0}^{0}(I+O(q x))(q x)^{T_{0}} C_{0} .
$$

Therefore, taking the limit $q \rightarrow 0$, we have

$$
\lim _{q \rightarrow 0}\left(\begin{array}{cc}
q^{-c_{0} / 2} & 0 \\
0 & q^{c_{0} / 2}
\end{array}\right) Y_{0}(q x)=\left(\begin{array}{cc}
l & 0 \\
0 & l^{-1}
\end{array}\right) x^{T_{0}} C_{0} .
$$

Comparing (1,1)-component of (58) and (59) at $x=\infty$, we obtain

$$
C_{0}=M_{\infty}, \quad l=\xi_{0}^{1 / 2}=\frac{c_{0} k}{p_{0}+c_{0} / 2} .
$$

By the latter half part of Proposition 3.1, we can conclude that $C_{0}=M_{\infty}$ is also independent of $s$.

\section{SPECIAL SOLUTIONS AND THEIR ASYMPTOTIC BEHAVIOR}

In this section, we find explicit solutions to the MPD system on elliptic curves for special values of parameters where the MPD system is linearizable. In the rational case, such a kind of special solutions are known as the Riccati solutions. For example, in the $P_{\mathrm{VI}^{-}}$-case, the Riccati solutions are solved in terms of Gauss's hypergeometric function. It is also known that the hypergeometric function has Euler's integral representation. Therefore, we may expect that our special solutions have some kind of integral representation. We show that our solutions can be written by the following integrals: for $i=1,2$,

$$
\int_{\gamma} e^{-2 \pi \sqrt{-1} c_{0} w} \vartheta_{1}(w)^{-c_{1}} \vartheta_{1}(w-t)^{c_{1}} \mathfrak{s}\left(w-t_{i} ; \lambda\right) d w,
$$

where we set $t_{1}=0, t_{2}=t$. We call this type of integrals Riemann-Wirtinger integrals. ${ }^{11,12,18}$ In the latter part of this section, applying Theorem 3.1 to our special solutions, we prove that the Riemann-Wirtinger integrals converge to the hypergeometric integrals as $q \rightarrow 0$.

We consider the case where $c_{1}+c_{2}=0$ for $c_{1} \notin \mathbb{Z}$ and $\gamma_{1}=\gamma_{2}=0$ on (8),

$$
\begin{gathered}
\frac{d Y}{d z}=A(z) Y, \\
A(z)=\left(\begin{array}{cc}
\alpha_{0}+\alpha_{1} \rho(z)-\alpha_{1} \rho(z-t) & \beta_{1} \mathfrak{s}(z ; \lambda)+\beta_{2} \mathfrak{s}(z-t ; \lambda) \\
0 & -\alpha_{0}-\alpha_{1} \rho(z)+\alpha_{1} \rho(z-t)
\end{array}\right) .
\end{gathered}
$$

Then the MPD systems (20)-(33) reduce to

$$
\begin{gathered}
\frac{\partial \lambda}{\partial t}=-2 \alpha_{1}, \quad \frac{\partial \alpha_{0}}{\partial t}=0, \quad \frac{\partial \alpha_{1}}{\partial t}=0, \\
\frac{\partial \beta_{1}}{\partial t}=-2 \pi \sqrt{-1} \alpha_{1} \beta_{1}-2 \alpha_{1} \beta_{1} \rho(t)-2 \alpha_{1} \beta_{2} \mathfrak{s}(t ;-\lambda),
\end{gathered}
$$




$$
\frac{\partial \beta_{2}}{\partial t}=-2 \pi \sqrt{-1} \alpha_{1} \beta_{2}+2 \alpha_{0} \beta_{2}+2 \alpha_{1} \beta_{2} \rho(t)+2 \alpha_{1} \beta_{1} \mathfrak{s}(t ; \lambda)
$$

and

$$
\begin{gathered}
2 \pi \sqrt{-1} \frac{\partial \lambda}{\partial \tau}=2 \alpha_{0}, \quad \frac{\partial \alpha_{0}}{\partial \tau}=0, \quad \frac{\partial \alpha_{1}}{\partial \tau}=0, \\
2 \pi \sqrt{-1} \frac{\partial \beta_{1}}{\partial \tau}=2 \alpha_{1} \beta_{2} \frac{\partial \mathfrak{s}}{\partial \lambda}(t ;-\lambda)+\alpha_{1} \beta_{1}\left(2 \wp(\lambda)-\rho(t)^{2}+\wp(t)\right), \\
2 \pi \sqrt{-1} \frac{\partial \beta_{2}}{\partial \tau}=-2 \alpha_{1} \beta_{1} \frac{\partial \mathfrak{s}}{\partial \lambda}(t ; \lambda)-\alpha_{1} \beta_{2}\left(2 \wp(\lambda)-\rho(t)^{2}+\wp(t)\right) .
\end{gathered}
$$

By (61) and (64), we can set

$$
\alpha_{0}=\pi \sqrt{-1} c_{0}, \quad \alpha_{1}=c_{1} / 2
$$

and

$$
\lambda=c_{0} \tau+c_{1} t-c_{\infty}
$$

for some constants $c_{0}, c_{\infty}$.

Proposition 4.1: Equation (60) is solvable. We have a fundamental system of solutions to (60) written as follows:

$$
Y(z)=\left(\begin{array}{cc}
y_{11} & e^{\pi \sqrt{-1} c_{1} t} y_{11}\left(\beta_{1} \int_{0}^{z} \Phi(w) d w+\beta_{2} \int_{0}^{z} \Psi(w) d w\right) \\
0 & y_{11}^{-1}
\end{array}\right),
$$

where

$$
\begin{gathered}
y_{11}=e^{\pi \sqrt{-1}\left(c_{0} z-\left(c_{1} / 2\right) t\right)} \vartheta_{1}(z)^{c_{1} / 2} \vartheta_{1}(z-t)^{-c_{1} / 2}, \\
\Phi(w)=e^{-2 \pi \sqrt{-1} c_{0} w} \vartheta_{1}(w)^{-c_{1}} \vartheta_{1}(w-t)^{c_{1}} \mathfrak{s}(w ; \lambda), \\
\Psi(w)=e^{-2 \pi \sqrt{-1} c_{0} w} \vartheta_{1}(w)^{-c_{1}} \vartheta_{1}(w-t)^{c_{1}} \mathfrak{s}(w-t ; \lambda) .
\end{gathered}
$$

Proof: Put $Y=\left(\begin{array}{l}y_{1} \\ y_{2}\end{array}\right)$, then $y_{2}$ must satisfy the following first-order linear differential equation:

$$
\frac{d y_{2}}{d z}=\left(-\pi \sqrt{-1} c_{0}-\frac{c_{1}}{2} \rho(z)+\frac{c_{1}}{2} \rho(z-t)\right) y_{2} .
$$

It is solved by

$$
y_{2}=d_{2} e^{-\pi \sqrt{-1}\left(c_{0} z-\left(c_{1} / 2\right) t\right)} \vartheta_{1}(z)^{-c_{1} / 2} \vartheta_{1}(z-t)^{c_{1} / 2}=d_{2} y_{11}^{-1},
$$

where $d_{2}$ is an integration constant. Then $y_{1}$ must satisfy the following first-order nonhomogeneous linear differential equation:

$$
\frac{d y_{1}}{d z}=\left(\pi \sqrt{-1} c_{0}+\frac{c_{1}}{2} \rho(z)-\frac{c_{1}}{2} \rho(z-t)\right) y_{1}+\left(\beta_{1} \mathfrak{s}(z ; \lambda)+\beta_{2} \mathfrak{s}(z-t ; \lambda)\right) y_{2} .
$$

It is solved by 


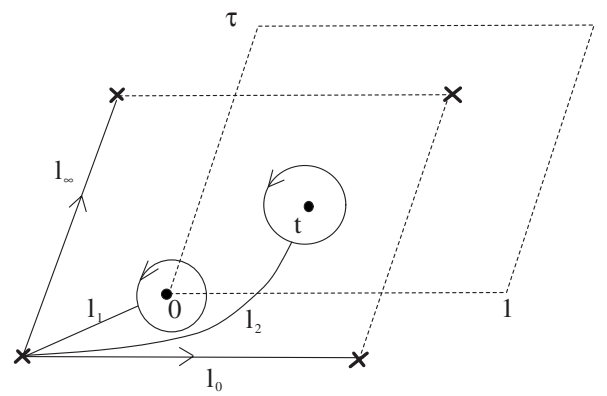

FIG. 1. Loops on $E_{\tau}$.

$$
y_{1}=d_{1} y_{11}+y_{11} \int_{0}^{z}\left(\beta_{1} \mathfrak{s}(w ; \lambda)+\beta_{2} \mathfrak{s}(w-t ; \lambda)\right) y_{2}(w) y_{11}(w)^{-1} d w,
$$

where $d_{1}$ is an integration constant. Hence we obtain the fundamental system of solutions (69).

We shall compute the monodromy matrices of $Y(z)$ along the loops drawn in Fig. 1. As for $l_{0}$ and $l_{\infty}$, we have

$$
Y^{l_{0}}(z)=Y(z) M_{0}, \quad Y^{l_{\infty}}(z)=\left(\begin{array}{cc}
e^{\pi \sqrt{-1} \lambda} & 0 \\
0 & e^{-\pi \sqrt{-1} \lambda}
\end{array}\right) Y(z) M_{\infty}
$$

where

$$
M_{0}=\left(\begin{array}{cc}
e^{\pi \sqrt{-1}} c_{0} & e^{\pi \sqrt{-1} c_{0}} N_{0} \\
0 & e^{-\pi \sqrt{-1} c_{0}}
\end{array}\right), \quad M_{\infty}=\left(\begin{array}{cc}
e^{\pi \sqrt{-1} c_{\infty}} & e^{\pi \sqrt{-1} c_{\infty}} N_{\infty} \\
0 & e^{-\pi \sqrt{-1} c_{\infty}}
\end{array}\right),
$$

and $Y(z)$ can be expanded at $z=0$ and $t$ as follows:

$$
\begin{gathered}
Y(z)=\left(\begin{array}{cc}
g_{11}^{(1)} & c_{1}^{-1} \beta_{1} g_{11}^{(1)-1} \\
0 & g_{11}^{(1)-1}
\end{array}\right)(I+O(z)) z^{T_{1}} \quad \text { at } \quad z=0, \\
=\left(\begin{array}{cc}
g_{11}^{(2)} & -c_{1}^{-1} \beta_{2} g_{11}^{(2)-1} \\
0 & g_{11}^{(2)-1}
\end{array}\right)(I+O(z-t))(z-t)^{-T_{1}} C_{2} \quad \text { at } z=t,
\end{gathered}
$$

where

$$
g_{11}^{(1)}=e^{-\pi \sqrt{-1} c_{1} t / 2} \vartheta_{1}^{\prime c_{1} / 2} \vartheta_{1}(-t)^{-c_{1} / 2}, \quad g_{11}^{(2)}=e^{\pi \sqrt{-1}\left(c_{0}-c_{1} / 2\right) t} \vartheta_{1}^{\prime-c_{1} / 2} \vartheta_{1}(t)^{c_{1} / 2},
$$

and $C_{2}=\left(\begin{array}{cc}1 & N_{2} \\ 0 & 1\end{array}\right)$. Here we define $N_{j}$ for $j=0,2, \infty$ by

$$
N_{j}=\beta_{1} \int_{\gamma_{j}} \Phi(w) d w+\beta_{2} \int_{\gamma_{j}} \Psi(w) d w,
$$

and the paths of integrals $\gamma_{j}(j=0,2, \infty)$ are given in Fig. 2. Hence we obtain the monodromy matrices along the loops $l_{1}$ and $l_{2}$,

$$
Y^{l_{1}}(z)=Y(z) M_{1}, \quad Y^{l_{2}}(z)=Y(z) M_{2},
$$

where $M_{1}, M_{2}$ are given by

$$
M_{1}=\left(\begin{array}{cc}
e^{\pi \sqrt{-1} c_{1}} & 0 \\
0 & e^{-\pi \sqrt{-1} c_{1}}
\end{array}\right),
$$




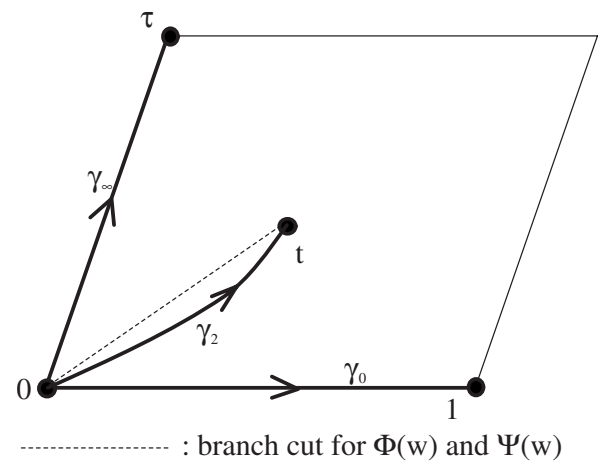

FIG. 2. Paths of integral.

$$
M_{2}=\left(\begin{array}{cc}
1 & N_{2} \\
0 & 1
\end{array}\right)^{-1}\left(\begin{array}{cc}
e^{-\pi \sqrt{-1} c_{1}} & 0 \\
0 & e^{\pi \sqrt{-1} c_{1}}
\end{array}\right)\left(\begin{array}{cc}
1 & N_{2} \\
0 & 1
\end{array}\right) .
$$

We have a unique relation among the generators $l_{0}, l_{1}, l_{2}, l_{\infty}$ of the fundamental group of $E_{\tau}$ and, therefore, we have a corresponding relation among the monodromy matrices $M_{0}, M_{1}, M_{2}, M_{\infty}$,

$$
M_{\infty}^{-1} M_{0}^{-1} M_{\infty} M_{0}=M_{1} M_{2} .
$$

Substituting (72), (74), and (75) into (76), we have

$$
\left(1-e^{-2 \pi \sqrt{-1} c_{\infty}}\right) N_{0}+\left(e^{-2 \pi \sqrt{-1}} c_{0}-1\right) N_{\infty}=\left(1-e^{2 \pi \sqrt{-1}} c_{1}\right) N_{2} .
$$

On the other hand, from the homotopy equivalence relation among the paths of integrals $\gamma_{0}, \gamma_{2}$, $\gamma_{\infty}$, we have

$$
\left(1-e^{-2 \pi \sqrt{-1} c_{\infty}}\right) \gamma_{0}+\left(e^{-2 \pi \sqrt{-1} c_{0}}-1\right) \gamma_{\infty}=\left(1-e^{2 \pi \sqrt{-1}} c_{1}\right) \gamma_{2} .
$$

Making use of the above computations on the monodromy matrices, we can solve the reduced MPD system (61)-(66).

Theorem 4.1: Assume that $c_{1} \notin \mathbb{Z}$. We put $\lambda=c_{0} \tau+c_{1} t-c_{\infty}$ and

$$
\beta^{(k)}=\left(\begin{array}{c}
\beta_{1}^{(k)} \\
\beta_{2}^{(k)}
\end{array}\right)=\left(\begin{array}{c}
-e^{2 \pi \sqrt{-1}\left(c_{0}-c_{1}\right) t} \int_{\gamma_{k}} \Psi(w) d w \\
e^{2 \pi \sqrt{-1}\left(c_{0}-c_{1}\right) t} \int_{\gamma_{k}} \Phi(w) d w
\end{array}\right)
$$

for $k=0,2, \infty$. Then $\beta^{(k)}(k=0,2, \infty)$ satisfy the following system of linear differential equations:

$$
\begin{gathered}
\frac{\partial \beta_{1}}{\partial t}=-(\pi \sqrt{-1}+\rho(t)) c_{1} \beta_{1}-c_{1} \mathfrak{s}(t ;-\lambda) \beta_{2}, \\
\frac{\partial \beta_{2}}{\partial t}=\left(\pi \sqrt{-1}\left(2 c_{0}-c_{1}\right)+c_{1} \rho(t)\right) \beta_{2}+c_{1} \mathfrak{s}(t ; \lambda) \beta_{1}, \\
4 \pi \sqrt{-1} \frac{\partial \beta_{1}}{\partial \tau}=2 c_{1} \frac{\partial \mathfrak{s}}{\partial \lambda}(t ;-\lambda) \beta_{2}+c_{1}\left(2 \wp(\lambda)-\rho(t)^{2}+\wp(t)\right) \beta_{1},
\end{gathered}
$$




$$
4 \pi \sqrt{-1} \frac{\partial \beta_{2}}{\partial \tau}=-2 c_{1} \frac{\partial \mathfrak{s}}{\partial \lambda}(t ; \lambda) \beta_{1}-c_{1}\left(2 \wp(\lambda)-\rho(t)^{2}+\wp(t)\right) \beta_{2}
$$

The solution space of the system (79)-(82) is of two dimensions. Three of the solutions $\beta^{(k)}, k$ $=0,2, \infty$, obey the unique linear relation

$$
\left(1-e^{-2 \pi \sqrt{-1} c_{\infty}}\right) \beta^{(0)}+\left(e^{-2 \pi \sqrt{-1} c_{0}}-1\right) \beta^{(\infty)}=\left(1-e^{2 \pi \sqrt{-1}} c_{1}\right) \beta^{(2)} .
$$

Proof: We have obtained the following correspondence between the coefficients of the differential equations (60) and the monodromy data of (60),

$$
\left(\begin{array}{l}
N_{\infty} \\
N_{0} \\
N_{2}
\end{array}\right)=\left(\begin{array}{ll}
\int_{\gamma_{\infty}} \Phi(w) d w & \int_{\gamma_{\infty}} \Psi(w) d w \\
\int_{\gamma_{0}} \Phi(w) d w & \int_{\gamma_{0}} \Psi(w) d w \\
\int_{\gamma_{2}} \Phi(w) d w & \int_{\gamma_{2}} \Psi(w) d w
\end{array}\right)\left(\begin{array}{l}
\beta_{1} \\
\beta_{2}
\end{array}\right) .
$$

Since the relation (77) is unique, two of $N_{k}$ 's can be taken freely. It means that the $3 \times 2$ matrix in the right hand side is of rank two. Particularly, the square matrix,

$$
F=\left(\begin{array}{ll}
\int_{\gamma_{\infty}} \Phi(w) d w & \int_{\gamma_{\infty}} \Psi(w) d w \\
\int_{\gamma_{0}} \Phi(w) d w & \int_{\gamma_{0}} \Psi(w) d w
\end{array}\right),
$$

is invertible by the assumption $c_{1} \notin \mathbb{Z}$. Hence we have

$$
\left(\begin{array}{l}
\beta_{1} \\
\beta_{2}
\end{array}\right)=F^{-1}\left(\begin{array}{c}
N_{\infty} \\
N_{0}
\end{array}\right),
$$

which means that

$$
\left(\begin{array}{c}
\beta_{1}^{(k)} \\
\beta_{2}^{(k)}
\end{array}\right)=\operatorname{det} F^{-1}\left(\begin{array}{c}
-\int_{\gamma_{k}} \Psi(w) d w \\
\int_{\gamma_{k}} \Phi(w) d w
\end{array}\right), \quad k=\infty, 0,
$$

form a system of fundamental solutions to the system of linear differential equations (79)-(82). On the other hand, $\operatorname{det}\left(\begin{array}{cc}\beta_{1}^{(\infty)} & \beta_{1}^{(0)} \\ \beta_{2}^{(\infty)} & \beta_{2}^{(0)}\end{array}\right)=\operatorname{det} F^{-1}$ satisfies the following differential equations:

$$
\begin{gathered}
\frac{\partial}{\partial t} \operatorname{det} F^{-1}=2 \pi \sqrt{-1}\left(c_{0}-c_{1}\right) \operatorname{det} F^{-1} \\
\frac{\partial}{\partial \tau} \operatorname{det} F^{-1}=0
\end{gathered}
$$

So we may take det $F^{-1}=e^{2 \pi \sqrt{-1}\left(c_{0}-c_{1}\right) t}$. The linear relation (83) follows from (78).

Next, we shall describe the behavior of the solutions as $q \rightarrow 0$. The differential system (60) tends to the following system on $\mathrm{P}^{1}(\mathrm{C})$ as shown in Sec. III: 


$$
\frac{d Y_{0}}{d x}=A^{0}(x) Y_{0}
$$

where

$$
A^{0}(x)=\frac{1}{x}\left(\begin{array}{cc}
c_{0} / 2 & 0 \\
0 & -c_{0} / 2
\end{array}\right)+\frac{1}{x-1}\left(\begin{array}{cc}
c_{1} / 2 & \beta_{1}^{0} \\
0 & -c_{1} / 2
\end{array}\right)+\frac{1}{x-s}\left(\begin{array}{cc}
-c_{1} / 2 & \beta_{2}^{0} \\
0 & c_{1} / 2
\end{array}\right) .
$$

Proposition 4.2: Equation (84) is solvable, whose fundamental solution is given by

$$
Y_{0}(x)=\left(\begin{array}{cc}
y_{0,11} & y_{0,11}\left(\beta_{1}^{0} \int_{1}^{x} \Phi_{0}(w) d w+\beta_{2}^{0} \int_{1}^{x} \Psi_{0}(w) d w\right) \\
0 & y_{0,11}^{-1}
\end{array}\right),
$$

where

$$
y_{0,11}=x^{c_{0} / 2}(x-1)^{c_{1} / 2}(x-s)^{-c_{1} / 2}
$$

and

$$
\Phi_{0}(w)=w^{-c_{0}}(w-s)^{c_{1}}(w-1)^{-c_{1}-1}, \quad \Psi_{0}(w)=w^{-c_{0}}(w-s)^{c_{1}-1}(w-1)^{-c_{1}} .
$$

Proof: The statement can be proven in the similar way to Proposition 4.1. Here we remark that the lower end 1 of the integrals in (85) corresponds to the lower end 0 in (69) because we adopt the coordinate $x=e^{2 \pi \sqrt{-1} z}$ on the rational curve.

We can expand the solution $Y_{0}(x)$ as follows:

$$
\begin{gathered}
Y_{0}(x)=G\left(I+O\left(x^{-1}\right)\right) x^{T_{0}} C_{\infty} \quad \text { at } x=\infty \\
=G_{1}^{0}(I+O(x-1))(x-1)^{T_{1}} \text { at } x=1 \\
=G_{2}^{0}(I+O(x-s))(x-s)^{-T_{1}}\left(\begin{array}{cc}
1 & N_{2} \\
0 & 1
\end{array}\right) \text { at } x=s \\
=G_{0}^{0}(I+O(x)) x^{T_{0}} C_{\infty}\left(\begin{array}{cc}
e^{\pi \sqrt{-1}} c_{\infty} & e^{\pi \sqrt{-1} c_{\infty}} N_{\infty} \\
0 & e^{-\pi \sqrt{-1} c_{\infty}}
\end{array}\right) \text { at } x=0,
\end{gathered}
$$

where

$$
\begin{gathered}
C_{\infty}=\left(\begin{array}{cc}
1 & \left(1-e^{-2 \pi \sqrt{-1} c_{0}}\right)^{-1} N_{0} \\
0 & 1
\end{array}\right), \\
G=\left(\begin{array}{cc}
1 & -c_{0}^{-1}\left(\beta_{1}^{0}+\beta_{2}^{0}\right) \\
0 & 1
\end{array}\right), \\
G_{1}^{0}=\left(\begin{array}{cc}
(1-s)^{-c_{1} / 2} & c_{1}^{-1} \beta_{1}^{0}(1-s)^{c_{1} / 2} \\
0 & (1-s)^{c_{1} / 2}
\end{array}\right), \\
G_{2}^{0}=\left(\begin{array}{cc}
s^{c_{0} / 2}(s-1)^{c_{1} / 2} & -c_{1}^{-1} \beta_{2}^{0} s^{-c_{0} / 2}(s-1)^{-c_{1} / 2} \\
0 & s^{-c_{0} / 2}(s-1)^{-c_{1} / 2}
\end{array}\right),
\end{gathered}
$$




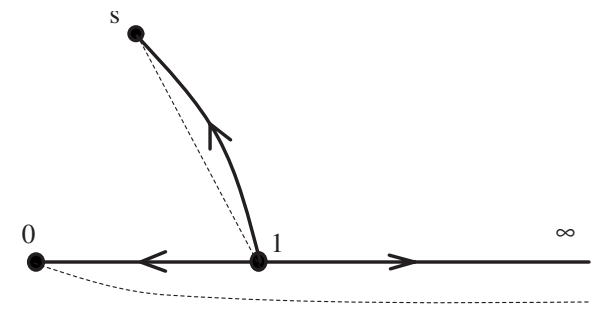

: branch cut for $\Phi_{0}(\mathrm{w})$ and $\Psi_{0}(\mathrm{w})$

FIG. 3. Paths of integral.

$$
G_{0}^{0}=\left(\begin{array}{cc}
e^{-\pi \sqrt{-1} c_{\infty}} S^{-c_{1} / 2} & 0 \\
0 & e^{\pi \sqrt{-1} c_{\infty} s_{1} / 2}
\end{array}\right),
$$

and

$$
\begin{gathered}
\beta_{1}^{0} \int_{1}^{\infty} \Phi_{0}(w) d w+\beta_{2}^{0} \int_{1}^{\infty} \Psi_{0}(w) d w=\left(1-e^{-2 \pi \sqrt{-1} c_{0}}\right)^{-1} N_{0}, \\
\beta_{1}^{0} \int_{1}^{s} \Phi_{0}(w) d w+\beta_{2}^{0} \int_{1}^{s} \Psi_{0}(w) d w=N_{2}, \\
\beta_{1}^{0} \int_{1}^{0} \Phi_{0}(w) d w+\beta_{2}^{0} \int_{1}^{0} \Psi_{0}(w) d w=N_{\infty}+e^{-2 \pi \sqrt{-1}} c_{\infty}\left(1-e^{-2 \pi \sqrt{-1} c_{0}}\right)^{-1} N_{0},
\end{gathered}
$$

the paths of integrals are given in Fig. 3. Applying Theorem 3.1, we obtain formulas on a relationship between the Riemann-Wirtinger integrals and the hypergeometric integrals.

Theorem 4.2: Assume that $0<\mathfrak{R} c_{0}<1$. We put

$$
\begin{gathered}
\Phi(w)=e^{-2 \pi \sqrt{-1} c_{0} w} \vartheta_{1}(w)^{-c_{1}} \vartheta_{1}(w-t)^{c_{1}} \mathfrak{s}(w ; \lambda), \\
\Psi(w)=e^{-2 \pi \sqrt{-1} c_{0} w} \vartheta_{1}(w)^{-c_{1}} \vartheta_{1}(w-t)^{c_{1}} \mathfrak{s}(w-t ; \lambda), \\
\Phi_{0}(w)=w^{-c_{0}}(w-s)^{c_{1}}(w-1)^{-c_{1}-1}, \\
\Psi_{0}(w)=w^{-c_{0}}(w-s)^{c_{1}-1}(w-1)^{-c_{1}},
\end{gathered}
$$

and $s=e^{2 \pi \sqrt{-1} t}, \quad q=e^{2 \pi \sqrt{-1} \tau}$. For any simply connected relatively compact open set $V \subset \mathbb{P}^{1}(C) \backslash\{0,1, \infty\}$, the Riemann-Wirtinger integrals converge uniformly on $s \in V$ to the hypergeometric integrals as follows:

$$
\begin{aligned}
& \lim _{q \rightarrow 0} \int_{0}^{1} \Psi(w) d w=\left(1-e^{-2 \pi \sqrt{-1} c_{0}}\right) \int_{1}^{\infty} \Psi_{0}(w) d w, \\
& \lim _{q \rightarrow 0} \int_{0}^{1} \Phi(w) d w=\left(1-e^{-2 \pi \sqrt{-1} c_{0}}\right) \int_{1}^{\infty} \Phi_{0}(w) d w,
\end{aligned}
$$




$$
\begin{gathered}
\lim _{q \rightarrow 0} \int_{0}^{t} \Psi(w) d w=\int_{1}^{s} \Psi_{0}(w) d w, \\
\lim _{q \rightarrow 0} \int_{0}^{t} \Phi(w) d w=\int_{1}^{s} \Phi_{0}(w) d w, \\
\lim _{q \rightarrow 0} \int_{0}^{\tau} \Psi(w) d w=\int_{1}^{0} \Psi_{0}(w) d w-e^{-2 \pi \sqrt{-1}} c_{\infty} \int_{1}^{\infty} \Psi_{0}(w) d w, \\
\lim _{q \rightarrow 0} \int_{0}^{\tau} \Phi(w) d w=\int_{1}^{0} \Phi_{0}(w) d w-e^{-2 \pi \sqrt{-1}} c_{\infty} \int_{1}^{\infty} \Phi_{0}(w) d w .
\end{gathered}
$$

Proof: From the relations (86)-(88), we have

$$
\left(\begin{array}{l}
N_{\infty} \\
N_{0} \\
N_{2}
\end{array}\right)=\left(\begin{array}{cc}
\int_{1}^{0} \Phi_{0}(w) d w-e^{-2 \pi \sqrt{-1}} c_{\infty} \int_{1}^{\infty} \Phi_{0}(w) d w & \int_{1}^{0} \Psi_{0}(w) d w-e^{-2 \pi \sqrt{-1}} c_{\infty} \int_{1}^{\infty} \Psi_{0}(w) d w \\
\left(1-e^{-2 \pi \sqrt{-1} c_{0}}\right) \int_{1}^{\infty} \Phi_{0}(w) d w & \left(1-e^{\left.-2 \pi \sqrt{-1} c_{0}\right)} \int_{1}^{\infty} \Psi_{0}(w) d w\right. \\
\int_{1}^{s} \Phi_{0}(w) d w & \int_{1}^{s} \Psi_{0}(w) d w
\end{array}\right)\left(\begin{array}{c}
\beta_{1}^{0} \\
\beta_{2}^{0}
\end{array}\right) .
$$

By a similar discussion in the proof of Theorem 4.1, we have

$$
\begin{aligned}
\left(\begin{array}{l}
\beta_{1}^{0} \\
\beta_{2}^{0}
\end{array}\right)= & s^{\left(c_{0}-c_{1}\right)}\left(\begin{array}{cc}
\left(1-e^{-2 \pi \sqrt{-1} c_{0}}\right) \int_{1}^{\infty} \Psi_{0}(w) d w & -\left(\int_{1}^{0} \Psi_{0}(w) d w-e^{-2 \pi \sqrt{-1} c_{\infty}} \int_{1}^{\infty} \Psi_{0}(w) d w\right) \\
-\left(1-e^{-2 \pi \sqrt{-1} c_{0}}\right) \int_{1}^{\infty} \Phi_{0}(w) d w & \int_{1}^{0} \Phi_{0}(w) d w-e^{-2 \pi \sqrt{-1} c_{\infty}} \int_{1}^{\infty} \Phi_{0}(w) d w
\end{array}\right) \\
& \times\left(\begin{array}{c}
N_{\infty} \\
N_{0}
\end{array}\right) .
\end{aligned}
$$

Applying Theorem 3.1, we obtain

$$
\begin{aligned}
\beta^{(0)} \rightarrow \beta_{0}^{(0)}= & \left(\begin{array}{l}
-s^{\left(c_{0}-c_{1}\right)}\left(1-e^{\left.-2 \pi \sqrt{-1} c_{0}\right)} \int_{1}^{\infty} \Psi_{0}(w) d w\right. \\
s^{\left(c_{0}-c_{1}\right)}\left(1-e^{\left.-2 \pi \sqrt{-1} c_{0}\right)} \int_{1}^{\infty} \Phi_{0}(w) d w\right.
\end{array}\right), \\
\beta^{(\infty)} \rightarrow \beta_{0}^{(\infty)}= & \left(\begin{array}{l}
-s^{\left(c_{0}-c_{1}\right)}\left(\int_{1}^{0} \Psi_{0}(w) d w-e^{-2 \pi \sqrt{-1} c_{\infty}} \int_{1}^{\infty} \Psi_{0}(w) d w\right) \\
s^{\left(c_{0}-c_{1}\right)}\left(\int_{1}^{0} \Phi_{0}(w) d w-e^{-2 \pi \sqrt{-1} c_{\infty}} \int_{1}^{\infty} \Phi_{0}(w) d w\right)
\end{array}\right),
\end{aligned}
$$

as $q \rightarrow 0$. In addition, since the relation (77) is preserved along the solutions to the MPD equation, we have 


$$
\left(1-e^{-2 \pi \sqrt{-1} c_{\infty}}\right) \beta_{0}^{(0)}+\left(e^{-2 \pi \sqrt{-1} c_{0}}-1\right) \beta_{0}^{(\infty)}=\left(1-e^{2 \pi \sqrt{-1}} c_{1}\right) \beta_{0}^{(2)},
$$

with

$$
\beta_{0}^{(2)}=\left(\begin{array}{c}
-s^{\left(c_{0}-c_{1}\right)} \int_{1}^{s} \Psi_{0}(w) d w \\
s^{\left(c_{0}-c_{1}\right)} \int_{1}^{s} \Phi_{0}(w) d w
\end{array}\right) .
$$

Hence we obtain $\beta^{(2)} \rightarrow \beta_{0}^{(2)}$ as $q \rightarrow 0$.

${ }^{1}$ Felder, G. and Varchenko, A., "Integral representation of solutions of the elliptic Knizhnik-Zamolodchikov-Bernard equations," Int. Math. Res. Notices 1995221 (1995).

${ }^{2}$ Hurwitz, A. and Courant, R., Vorlesungen Über Allgemeine Funktionentheorie und Elliptische Funktionen (SpringerVerlag, Berlin, 1964).

${ }^{3}$ Jimbo, M., "Monodromy problem and the boundary condition for some Painlevé equations," Publ. RIMS Kyoto Univ. 18, 1137 (1982).

${ }^{4}$ Jimbo, M., Miwa, T., and Ueno, K., "Monodromy preserving deformation of linear ordinary differential equations with rational coefficients," Physica D 2, 306 (1981).

${ }^{5}$ Jimbo, M. and Miwa, T., "Monodromy preserving deformation of linear ordinary differential equations with rational coefficients. II," Physica D 2, 407 (1981).

${ }^{6}$ Kawai, S., "Isomonodromic deformation of Fuchsian projective connections on elliptic curves," Nagoya Math. J. 171, 127 (2003)

${ }^{7}$ Korotkin, D., "Isomonodromic deformations in genus zero and one: algebro-geometric solutions and Schlesinger transformations. Integrable systems: From classical to quantum," CRM Proc. Lecture Notes 26, 87 (2000).

${ }^{8}$ Korotkin, D. and Samtleben, H., "On the quantization of isomonodromic deformations on the torus," Int. J. Mod. Phys. A 12, 2013 (1997)

${ }^{9}$ Kuroki, G. and Takebe, T., "Bosonization and integral representation of solutions of the Knizhnik-ZamolodchikovBernard equations," Commun. Math. Phys. 204, 587 (1999).

${ }^{10}$ Mano, T., "Monodromy Preserving Deformation of Linear Differential Equations on a Rational Nodal Curve" (submitted).

${ }^{11}$ Mano, T., "The Riemann-Wirtinger integral and monodromy-preserving deformation on elliptic curves," Int. Math. Res. Notices 2008 (2008), ID: rnn110.

${ }^{12}$ T. Mano, H. Watanabe, "Twisted cohomology and homology groups associated to the Riemann-Wirtinger integral" (submitted).

${ }^{13}$ Okamoto, K., “On Fuchs' problem on a torus. I," Funkc. Ekvac. 14, 137 (1971).

${ }^{14}$ Okamoto, K., "The Hamiltonian structure derived from the holonomic deformation of the linear ordinary differential equations on an elliptic curve," Sci. Pap. Coll. Arts Sci., Univ. Tokyo 37, 1 (1987).

${ }^{15}$ Iwasaki, K., Kimura, H., Shimomura, S., and Yoshida, M., From Gauss to Painlevé, Aspects of Mathematics (Vieweg, Braunschweig, 1991), Vol. E16.

${ }^{16}$ Sasaki, Y., "Special solutions of the Hamiltonian system on an elliptic curve," J. Math. Sci. Univ. Tokyo 9, 669 (2002).

${ }^{17}$ Sato, M., Miwa, T., and Jimbo, M., "Holonomic quantum fields. II: The Riemann-Hilbert problem," Publ. Res. Inst. Math. Sci. 15, 201 (1979)

${ }^{18}$ Riemann, B., in Nachträge, edited by Noether, M. and Wirtinger, W. (Teubner, Leipzig, 1902).

${ }^{19}$ Takasaki, K., "Gaudin model, KZ equation, and isomonodromic problem on torus," Lett. Math. Phys. 44, 143 (1998).

${ }^{20}$ Whittaker, E. T. and Watson, G. N., A Course of Modern Analysis (Cambridge University Press, London, 1902). 